\title{
A Pressure Management System for the Neurogenic Bladder After Spinal Cord Injury
}

\author{
E.J. McGuire, F. Noll, and F. Maynard \\ Section of Urology, Department of Surgery, and Department of Physical Medicine and \\ Rehabilitation, University of Michigan, Ann Arbor, Michigan
}

\begin{abstract}
One hundred eighteen patients consecutively admitted to a University Rehabilitation Program entered a protocol study of urologic management. Bladder pressures were kept below $30 \mathrm{cms} / \mathrm{H}_{2} \mathrm{O}$ by urologic treatment. Serial urodynamic, radiographic, bacteriologic, and endoscopic studies were performed at regular intervals. Eighteen patients have been lost to follow-up; 100 patients were followed for a mean 25.4 months with a range of 6 months to 56 months. There were 105 patients with lesions superior to the sacral segments and 13 patients with low lesions. At discharge 11 patients were voiding normally, 105 were continent on an intermittent catheterization (IC) protocol, and 2 patients used condom catheter drainage following sphincterotomy. Bacterial cultures and urinalysis data showed little or no relationship to clinical outcome, and treatment for 387 weeks by antimicrobial agents was not associated with discerniblc benefit as opposed to no treatment. Five patients developed bladder calculi, and five devcloped unilateral epididymitis. Bladder pressure was relatively easy to control following spinal cord injury, a result which suggests that high bladder pressure is not a direct result of the neural injury, but rather an evolutionary change as a result of bladder and urethral interactive dysfunction.
\end{abstract}

Key words: incontinence, urologic, urinary tract

\section{INTRODUCTION}

The urologic care of spinal cord injured individuals has evolved slowly over the past 20 years. Bors and Comar [1971], Gutman and Frankel [1966], and Pearman [1976] used a variety of methods in conjunction with early intermittent catheterization to achieve balanced bladder function in males, and if that failed sphincterotomy was employed to improve vesical emptying. The recognition that the effects of spinal cord injury on the lower urinary tract were complex and impacted on both low-pressure urinary storage and voiding function lead to efforts to improve bladder storage capacity or to preserve it [McGuire and Savastano 1985]. High, sustained intra-vesical pressure appears to be the most important cause of upper urinary tract complications

Received for publication December 18, 1990; accepted December 26, 1990.

Address reprint requests to E.J. McGuire, Section of Urology, University of Michigan Hospitals, 2916 Taubman Center, Ann Arbor, Michigan 48109-0330. 
TABLE I. Follow-Up Status of All Patients

\begin{tabular}{lcc}
\hline Months & Number & Lost to follow-up \\
\hline$>48$ & 7 & 2 \\
$>36$ & 30 & 4 \\
$>24$ & 38 & 6 \\
$>12$ & 31 & 4 \\
$>6$ & 12 & 2 \\
Mean 25.44 months & 118 & 18 \\
\hline
\end{tabular}

in patients with spinal cord injury and others with neurogenic bladder dysfunction [McGuire and Savastano, 1983; Wang et al., 1988]. Intravesical pressure is immediately related to bladder compliance and reflex contractility but ultimately to outlet resistance [Wang et al., 1989]. We set out to control intravesical pressure prospectively and to determine whether that would prevent the common urologic complications associated with spinal cord injury. Although we were able to control bladder pressure relatively easily in most instances by intermittent catheterization we did not initially intend to use that method as an exclusive management technique. The basic aim of the study was to simply determine the effectiveness of preservation of low bladder pressure and to determine the significance of the various urologic and urodynamic findings recorded in patients with spinal cord injury. Ultimately we intended to compare lower tract findings with the status of the upper urinary tract.

\section{Patient Population}

There were 61 patients with lesions between $\mathrm{C}_{1}$ and $\mathrm{C}_{7}$. Of these 31 were judged "complete" neural injuries and 26 were judged "incomplete." There were 44 patients with injuries between $T_{1}$ and $T_{12}, 27$ of these were judged complete, and 17 incomplete. There were 13 patients with lesions between $L_{1}$ and $L_{4}$ : four of these were judged complete and 9 incomplete. The mean age of all patients was 26 (range 18-74). Twenty-four patients were females, and ninety-four male. Follow-up after discharge is given in Table I. The mean elapsed time from injury to study entry was seven days with a range of 1-31 days.

\section{METHODS}

Upon entry to the study all patients underwent a fluoroscopically guided urodynamic evaluation consisting of the simultaneous measurement of bladder and external urethral sphincter pressure during filling to capacity or a bladder contraction. The study was done with $20 \%$ radio-opaque contrast material at $37^{\circ} \mathrm{C}$ with the bladder filled at medium rate. In addition an intravenous urogram or ultrasound study of the bladder and upper urinary tract was obtained at the initiation of the study as well as a cystoscopy and urinary cytology. During hospitalization on the rehabilitation unit, urodynamic studies consisting of simple pressure volume curves to those volumes attained at the time of intermittent catheterization were done monthly. All patients had a weekly urine analysis and urine culture. Urodynamic studies were repeated immediately for episodes of incontinence, presumed symptomatic urinary tract infection, or symptomatic autonomic dysreflexia. These studies usually consisted of a simple water cystometrogram (CMG) but if required for precise diagnosis a fluoroscopically guided study was done in conjunction with appropriate upper tract 
TABLE II. Level of Injury and Lower Tract Functional Classification

\begin{tabular}{llclcr}
\hline Status & Level & Number & Normal & $\begin{array}{c}\text { Reflex- } \\
\text { discoordinate }\end{array}$ & a-reflex \\
\hline Complete & $\mathrm{C}_{1}-\mathrm{C}_{7}$ & 35 & 0 & 32 & 3 \\
Incomplete & $\mathrm{C}_{1}-\mathrm{C}_{7}$ & 26 & 4 & 12 & 10 \\
Complete & $\mathrm{T}_{1}-\mathrm{T}_{12}$ & 27 & 0 & 20 & 7 \\
Incomplete & $\mathrm{T}_{1}-\mathrm{T}_{12}$ & 17 & 4 & $72(69 \%)$ & 5 \\
& & 105 & $8(8 \%)$ & 0 & $25(24 \%)$ \\
Complete & $\mathrm{L}_{1}-\mathrm{L}_{4}$ & 4 & $1^{\mathrm{a}}$ & 1 & 3 \\
Incomplete & $\mathrm{L}_{1}-\mathrm{L}_{4}$ & 9 & $5^{\mathrm{a}}$ & $1(8 \%)$ & $6(46 \%)$ \\
& & 13 & $6(46 \%)$ & & 3 \\
\hline
\end{tabular}

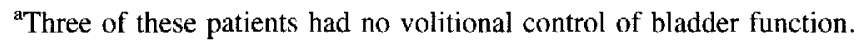

radiographic imaging. After discharge the same urodynamic studies were repeated at 3 month intervals and all entry studies repeated yearly.

At some time all patients were assigned a urologic diagnosis in one of the following categories: a reflex bladder with a coordinate sphincter, a reflex bladder with a discoordinate sphincter, or detrusor areflexia. Initially we used subheadings designating compliance values in the reflex and nonreflux groups, but since compliance was invariably normal we abandoned these. Patients classified as having bladder areflexia never, at any time, showed reflex contractile activity. Patients classified with the reflex bladder group showed a detrusor contraction at some time, even though treatment may have later produced bladder areflexia. Treatment was predicated on urodynamic findings but was directed towards the achievement of bladder pressure less than $20 \mathrm{~cm} / \mathrm{H}_{2} \mathrm{O}$ in all circumstances. Anticholinergic agents we initiated for control of incontinence, if that occurred, or if bladder pressures at volumes recovered by intermittent catheterization reached $20 \mathrm{~cm} / \mathrm{H}_{2} \mathrm{O}$. Initial therapy was Oxybutynin Chloride $5 \mathrm{mg}$ T.I.D.; if that was insufficient, Imipramine $10-25 \mathrm{mg}$ T.I.D. was added. If side effects prevented full use of oral agents or oral agents did not control bladder pressure, a solution of glycopyrolate in saline was instilled into the bladder after each catheterization (glycopyrolate $8 \mathrm{mg} / 500 \mathrm{ml}$ saline; $30 \mathrm{ml}$ instillation aliquot).

\section{RESULTS}

\section{Urodynamic Category of Bladder Function (Table II)}

Of 105 patients with lesions superior to the sacral cord segments, or those with injuries from $C_{1}$ through $T_{12}$, eight, all with incomplete lesions, regained normal reflex bladder function. Twenty-five of one hundred five never regained reflex bladder contractility. Of 62 patients with complete lesions 52 or $83 \%$ developed reflex bladder contractility with a discoordinate external sphincter, while 10 or $17 \%$ never regained reflex bladder contractility. Of 43 patients with incomplete lesions between $\mathrm{C}_{7}$ and $\mathrm{T}_{12} 8$ or $19 \%$ regained normal volitional reflex bladder and urethral function, 20 developed reflex bladder contractility with a discoordinate sphincter $(47 \%)$, and 15 never developed reflex bladder contractility (35\%). Thus of 105 patients with neural lesions traditionally associated with high-pressure reflex bladder dysfunction 
TABLE III. Status at Discharge

\begin{tabular}{lccccccc}
\hline & & \multicolumn{5}{c}{ Intermittent Cath. } \\
\cline { 3 - 7 } Level & Number & Normal & mo & 1 & 2 & 3 & \\
\hline $\mathrm{C}_{1}-\mathrm{T}_{12}$ & 105 & 8 & 25 & 36 & 25 & $10^{\mathrm{a}}$ & 1 condom \\
$\mathrm{L}_{\mathbf{1}}-\mathrm{L}_{4}$ & 13 & 3 & 6 & 3 & 0 & 0 & 1 condom \\
& 118 & $11(9.3)$ & $31(26)$ & $39(33)$ & $25(21)$ & $10(8)$ & \\
\hline
\end{tabular}

a Two patients in this group later failed and had an augmentation cytoplasty (1) and sphincterotomy (1).

and external sphincter dyssynergia $24 \%$ never regained any reflex bladder contractility, including $17 \%$ of those with complete lesions.

Of 13 patients with lesions between $\mathrm{L}_{1}$ and $\mathrm{L}_{4}$, three regained normal reflex and volitional bladder and urethral function, one developed reflex bladder contractility with a discoordinate sphincter, and six never regained reflex bladder contractility. Three patients in this group who recovered normal reflex bladder contractility (i.e., a coordinate relationship between bladder and external sphincter) were totally unable to control that activity and were incontinent. While $46 \%$ of those patients with lesions below $\mathrm{T}_{12}$ never developed reflex contractility, $54 \%$ did do so.

\section{Control of Bladder Pressure and Status at Discharge, and Follow-Up (Table III)}

Of the 105 patients with lesions at or above $T_{12}, 8$ regained normal bladder function while 25 never developed reflex bladder contractility. None of these patients required medication for control of bladder pressure. Of the remaining 72 patients, 36 or $50 \%$ required a single agent (oxybutynin chloride $5 \mathrm{mg}$ T.I.D.), 25 (35\%) required two agents (oxybutynin and impiramine $\mathrm{HCl} 10-25 \mathrm{mg} \mathrm{T.I.D.),} \mathrm{and} 10$ (9.5\%) required two oral agents and a third agent instilled directly into the bladder at the time of intermittent catheterization (glycopyrrolate solution $8 \mathrm{mg} / 500 \mathrm{ml}$ saline, $30 \mathrm{ml}$ each time). One patient had a sphincterotomy and was discharged using condom catheter drainage. Following discharge five patients became incontinent. This required the initiation of anticholinergic therapy in two, and the addition of another agent in three. One female patient, of the five, ultimately underwent an augmentation cystoplasty to control low-pressure reflex bladder contractility. One patient underwent an elective sphincterotomy and conversion to condom catheter drainage because he could not manage intermittent catheterization, and four patients had to be reminded to take their medication.

Of thirteen patients with lesions below $T_{12}$, three were voiding normally at the time of discharge, six used intermittent catheterization without medication, three used intermittent catheterization and one anticholinergic agent (oxybutynin or imipramine), and one used an external penile collecting device.

Of the entire group $9 \%$ regained normal bladder function, $26 \%$ used intermittent catheterization without any other treatment, and 72 or $63 \%$ used intermittent catheterization with anticholinergic agents. Two patients used condom catheter drainage. With the exception of these two and the eleven patients with normal bladder function, all other patients were continent at the time of discharge on an intermittent catheter- 
ization schedule interval of 4 to 6 hours. No patient had any abnormal findings by an upper tract imaging study, either intravenous urography or ultrasonography.

\section{Urologic Complications}

Stress incontinence. Five of twenty-four women developed symptomatic stress incontinence, as did one male patient. Of this group four had lesions at $T_{12}$ including the single male patient. The others had spinal lesions at $\mathrm{C}_{7}$ and $\mathrm{L}_{4}$, respectively. Of fourteen patients with lesions at $\mathrm{T}_{12}$, four developed stress incontinence requiring collagen therapy in one, and urethral suspension surgery in three.

Bladder calculi. Four patients developed bladder calculi in the first year after injury which were easily removed cystoscopically. Three of these occurred in tetraplegics, and one occurred in a patient with a $\mathrm{L}_{4}$ injury.

Epididymitis. There were five patients who developed symptomatic epididymitis which responded promptly to treatment.

Autonomic dysreflexia. Three tetraplegic patients had transient difficulty with autonomic dysreflexia. In two of these the dysreflexia was precipitated by a large bladder volume without any evidence of bladder reflex activity, but in one patient the dysreflexia was associated with small volume detrusor hyperreflexia.

Urinary incontinence. There were 35 episodes of reported urinary leakage, 11 of which occurred following discharge from the rehabilitation center. Of twenty-four episodes of urinary leakage occurring during initial rehabilitation, three were due to stress incontinence rather than reflex bladder contractility. In the remainder the mean bladder pressure at leakage was $55 \mathrm{~cm} / \mathrm{H}_{2} \mathrm{O}$. At the time of leakage urine cultures were positive for significant $\left(10^{5} \mathrm{~mm}^{3}\right)$ growth in sixteen instances, and negative in five. Following discharge leakage was reported by 11 patients. In seven instances urine cultures were positive, but in four urine cultures showed no growth. In three cases stress urinary incontinence was diagnosed in discharged patients by video urodynamic studies, and in the remainder reflex bladder contractility was revealed on a cystometrogram. Of these, two required the initiation of anticholinergic therapy, for the first time, three required a second agent, and the rest were encouraged to take medication which had been prescribed.

\section{Loss to Follow-Up (18 patients)}

Six of eleven patients who re-established normal voiding did not return for follow-up. Of those lost to follow-up who were managed by intermittent catheterization, one required no drugs, eight required one drug, and three required two drugs at the time of discharge. Of this group of twelve, five never returned for follow-up and the other seven returned, on the average, for 1.4 years prior to being lost to further follow-up.

\section{Bacteriologic Results Antimicrobial Therapy and Urine-Analysis Data}

Of 1,373 urine cultures, $459(33 \%)$ showed no growth. Despite the negative cultures, $6 \%$ of urine analyses done the same day showed significant pyuria $(>25$ wbc/hpf); 916 cultures showed some growth, with 659 showing colony counts greater than $10^{5}$ organisms $/ \mathrm{ml}$, and 257 cultures showing colony counts less than $10^{5}$ organisms $/ \mathrm{ml}$. All of these cultures were the product of catheterized urine specimens, and colony counts probably are not a valid measure of "contamination" versus true 
bacteriuria; thus $67 \%$ of cultures were positive and $33 \%$ negative. Twenty-two percent of the "positive" cultures were associated with pyuria ( $>25$ wbes/hpf).

Eighty-seven patients $(74 \%)$ received antimicrobial treatment at some time during hospitalization. In all, 387 weeks of antimicrobial therapy were given to this patient group. Antimicrobial therapy lead to an observed 309 weeks during which urine cultures showed no growth. Seventy-two patients received oral antimicrobial therapy, which lasted for 1 to 4 weeks. Twenty-seven such courses were associated with negative urine cultures the first week following cessation of therapy, and fortyfive with positive cultures. One-hundred twenty-eight weekly courses of direct instillation of an antimicrobial agent $480 \mathrm{mg}$ of gentamicin/liter saline instilled as a 30 $\mathrm{ml}$ aliquot after catheterization) were associated in $17 \%$ with negative cultures 1 week after cessation of therapy.

Twenty-nine patients were treated with parenteral antimicrobial agents for a presumed febrile urinary tract infection (temp 101 or $>$ ). Cultures plated immediately prior to initiation of therapy showed growth $68 \%$, and no growth in $32 \%$. Of this group, $70 \%$ were quadriplegics and $30 \%$ paraplegics.

Thirty-one patients never received antimicrobial agents during an average stay of 14.2 weeks. Four weeks were associated with a negative urine culture, and ten with a positive culture. Eighty-seven patients who received antimicrobial therapy had an average stay of 14.7 weeks, of which 3.5 were associated with a negative culture, and 11.2 with a positive culture.

\section{DISCUSSION}

The urodynamic results indicate that spinal cord injury has an effect on lower urinary tract function, which involves both the sphincter and bladder. Despite the number and preponderance of patients with what used to be called "upper motor neuron lesions," all but two of those patients who did not regain normal bladder function were discharged, continent on an intermittent catheterization protocol. To achieve adequate reservoir capability a combination of anticholinergic agents was required in approximately $70 \%$. However $25 \%$ of patients with high spinal cord injury never developed reflex bladder activity suggesting that bladder reflex activity is impaired by the spinal cord injury. Moreover the relative ease with which we were able to effectively stop reflex bladder contractility suggests that these are not hyperreflexic bladders as a result of the neural injury itself. We know from past experience reported by many workers that, with time, many patients with upper motor neuron lesions can develop very high pressure, sustained repetitive bladder contractility opposed by vigorous external sphincter activity [Perkash, 1978]. Despite an average follow-up of 24 months since discharge we have not actually encountered that kind of high-pressure hyper-reflexic bladder dysfunction. It may be that regular intermittent catheterization interrupts the interaction of bladder and external urethral sphincter that, with time, leads to progressive loss of bladder capacity, high intravesical pressures, and a requirement for a decrease in outlet resistance or some other method of treatment to effect better urinary drainage. While some of these patients could have been managed by sphincterotomy we did that only when we could not control incontinence or bladder pressures using drugs and intermittent catheterization. In one instance we used an augmentation cystoplasty to circumvent low-pressure reflex bladder contractility in a female patient with a $\mathrm{T}_{12}$ level spinal cord injury. Despite 
the number of quadriplegics who constitute over $50 \%$ of the study population we had difficulty with autonomic dysreflexia only in three patients. In two of these the dyreflexic episodes occurred at night in association with very large bladder volumes. They were treated by adjusting catheterization schedules. In the other patient, autonomic dysreflexia was related to reflex, low-volume, detrusor contractility and responded to anticholinergic agents. Bladder calculi were encountered in five patients and were easily removed at the time of cystoscopy. There were five cases of the unilateral epididymitis, which may be related to the high percentage of patients with chronic bacteriuria, and/or the intermittent catheterization.

The data collected regarding urinary tract infection are difficult to analyze. The number of positive cultures was so much greater than the actual number of patients who required parenteral antimicrobial agents for a presumed febrile urinary tract infection that it seems likely that neither a positive culture or pyuria are good indicators of the actual pathophysiologic process we wanted to identify. Disparity between culture and urinalysis data and clinical outcome is so great that the study of a given treatment to determine whether that treatment prevented infectious complications requires some indicator of efficacy other than presence or absence of bacteriuria. Despite 387 courses of antimicrobial therapy there was no lasting effect on the number of positive cultures nor was it clear that treatment of positive cultures and/or pyuria had any effect on continence, spasticity, pain, or the other symptoms, which are often attributed to urinary tract infections. It is equally difficult to determine whether continuous suppressive antimicrobial therapy had any influence on the development of febrile urinary tract infections. Anderson [1980] noted that the incidence of bacteriuria was decreased in a short-term study of spinal cord injured population treated by intermittent catheterization when prophylactic anticrobials were used. However only one patient in that studied group actually developed a febrile infection so that conclusions regarding efficacy drawn the study are difficult to accept. Ross and co-workers [1976] reported on the incidence of bacteriuria and upper urinary tract abnormalities in patients who underwent sphincterotomy over a 20 -year follow-up period. Of 112 patients, $27 \%$ were uninfected, $21 \%$ intermittently infected, and $50 \%$ always infected. The results of urine cultures in hospitalized patients in this study are almost identical; $50 \%$ virtually always positive, $20-30 \%$ negative, and $20-30 \%$ intermittently positive. The patient group in this report showed preservation of normal upper urinary tracts, and indeed improvement in the upper urinary tract over that recorded in the pre-operative situation. Only one patient of the one hundred twelve developed progressive hydroureteronephrosis and renal failure and his voiding pressures are not recorded. Putting our results in perspective with other reported series is difficult because the treatment we used was guided entirely by bladder pressure and there is no comparable series in the literature. Hackler [1977] reported that at 20 years, the incidence of abnormal pyelograms in patients treated by condom catheter drainage ranged from 25 to $40 \%$ and 36 to $52 \%$ in patients treated by catheters. Webb and co-workers (1984) reported a normal IVP in $91 \%$ of 406 spinal injury patients followed during a 15 -year period. Of that group $73 \%$ of those with complete lesions had a bladder outlet surgical procedure and in some $64 \%$ a secondary procedure was required. These procedures were based on residual urine volumes and intravenous urograms and not pressure data. Pearman [1976] reported $96 \%$ normal pyelogram rate in 99 patients followed for about the same length of time as patients in this report. We used a simple pressure management system to establish 
control of the lower urinary tract. Video urodynamics and other more sophisticated studies were not required nor is it likely that periodic upper urinary tract radiographic evaluation is essential provided bladder pressures remain controlled. It would appear that preservation of low bladder pressures can effectively reduce short-term urologic complications in patients with spinal cord injury.

The place of periodic urine cultures in the management of spinal cord injured patients is not clear. Of the 19 patients with a fever presumed to be urinary in origin precisely with same percentage of patients had a positive culture $(68 \%)$ as the general population without a fever. Moreover febrile episodes were much more common in quadriplegics than in paraplegics suggesting that factors other than bacteriuria were involved.

\section{ACKNOWLEDGMENTS}

This work was partially supported by funds from National Institute on Disability Research, Washington, D.C., grant G008535130.

\section{REFERENCES}

Bors E, Comar AE, (1971): "Neurological Urology.” Baltimore: University Park Press.

Guttman L, Frankel H (1966): The Value of Intermittent Catheterization in the Early Management of Traumatic Paraplegia and Paraplegia Tetraplegia. 4:63.

Pearman JW (1976): Urological follow-up of 99 spinal cord injured patients initially managed by intermittent catheterization. Br J Urol 48:297.

McGuire EJ, Savastano JA (1985): Urodynamics and management of the neuropathic bladder in spinal cord injury patients. J Am Paraplegia Soc 8:28.

McGuire EJ, Savastano JA (1983): Long term follow-up of spinal cord injury patients managed by intermittent catheterization. J Urol 129:775.

Webb DR, Fitzpatrick JM, O'Flynn JD (1984): A 15 year follow-up of 400 consecutive spinal cord injuries. Br J Urol 56:614.

Wang SC, McGuire EJ, Bloom DA (1988): A bladder pressure management system for myelodysplasia-Clinical outcome. J Urol 140:1499.

Wang SC, McGuire EJ, Bloom DA (1989): Urethral dilation in the management of urologic complications of myelodysplasia. J Urol 142:1054.

Perkash I (1978): Detrusor-sphincter dyssynergic responses. J Urol 120:469.

Anderson RV (1980): Prophylaxis of bacteriuria during intermittent catheterization of the acute neurogenic bladder. J Uro] 123:364.

Ross JC, Gibbon NOK, Sham GS (1976): Division of the external urethral sphincter in the neurogenic bladder: A 20 year review. Br J Urol 48:649.

Hackler RH (1977): A 25 year prospective mortality study in the spinal cord injured patient: Comparison with the long term living paraplegic. J Urol 117:486. 